Musées, Patrimoine et Culture scientifiques et techniques

$151 \mid 2014$

janvier-février 2014

\title{
L'Expérimentarium et les bibliothèques
}

\section{Élise Cellier-Holzem}

URL : http://journals.openedition.org/ocim/1418

DOI : $10.4000 /$ ocim. 1418

ISSN : 2108-646X

\section{Éditeur}

OCIM

Édition imprimée

Date de publication : 1 février 2014

ISSN : 0994-1908

\section{Référence électronique}

Élise Cellier-Holzem, "L'Expérimentarium et les bibliothèques », La Lettre de I'OCIM [En ligne], 151 | 2014, mis en ligne le 31 janvier 2015, consulté le 02 mai 2019. URL : http:// journals.openedition.org/ocim/1418; DOI : 10.4000/ocim.1418

Ce document a été généré automatiquement le 2 mai 2019.

Tous droits réservés 


\title{
L'Expérimentarium et les bibliothèques
}

\author{
Élise Cellier-Holzem
}

\section{L'Experimentarium}

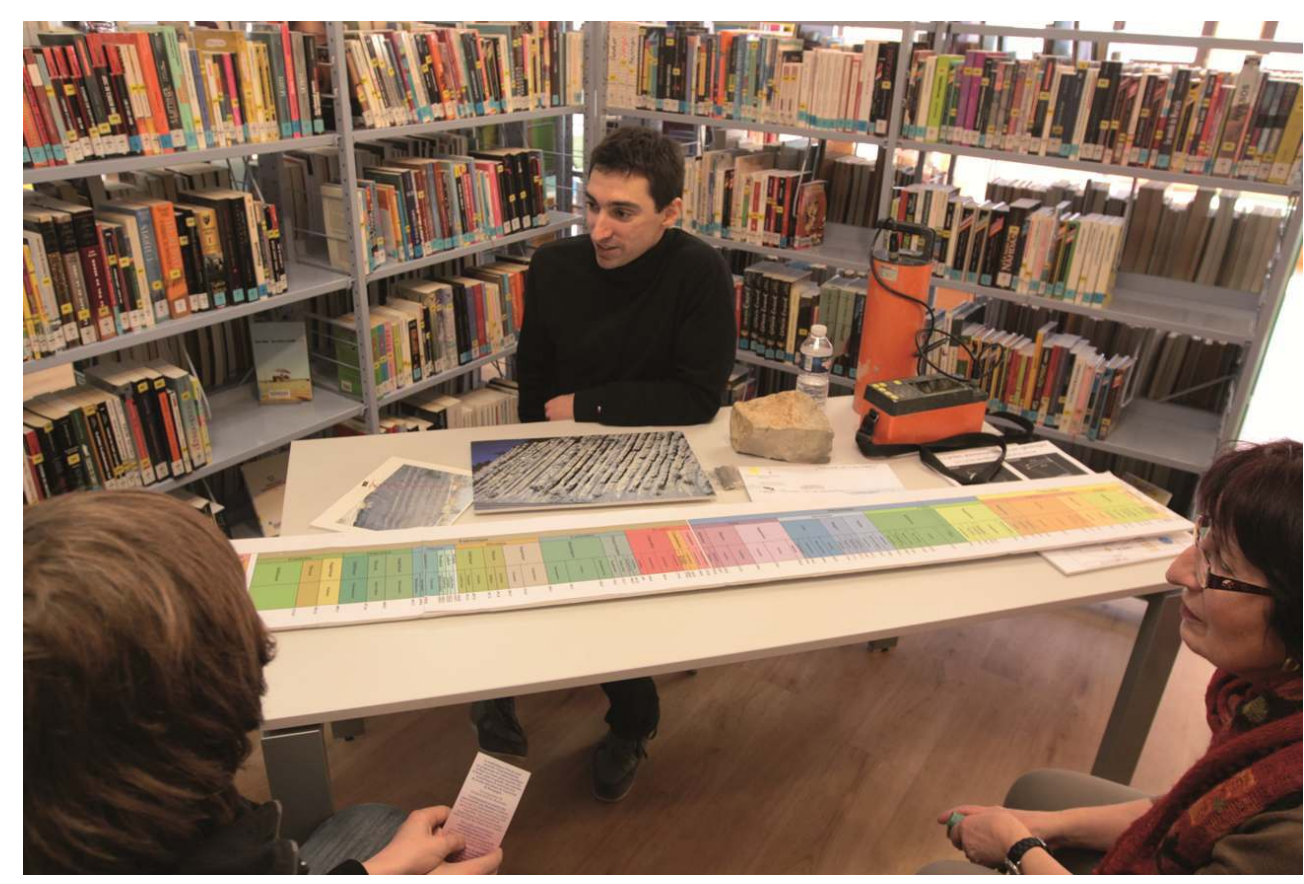

Mathieu Martinez présente son travail sur Les changements climatiques passés, enregistrés dans des roches à la bibliothèque du Port du Canal à Dijon (février 2012).

(c) Experimentarium

1 L'Experimentarium est un programme de la Mission Culture Scientifique de l'université de Bourgogne, créé en 2001. Il permet au public de rencontrer de jeunes chercheurs de l'université de Bourgogne dans un cadre que nous avons voulu sympathique et convivial. Autour d'expériences ou d'objets insolites, le chercheur raconte son quotidien, invite au 
questionnement et entraîne le visiteur au cœur de sa recherche. Il ne s'agit ni de professer sur des sujets scientifiques, ni de proposer des expériences didactiques. Les chercheurs parlent de leur vécu, partagent leurs connaissances et leur quotidien de recherche. Le fonctionnement mis en place (des ateliers-discussions d'une vingtaine de minutes et un environnement propice aux échanges) vise à créer un véritable dialogue et rendre l'échange le plus riche possible pour les visiteurs comme pour les chercheurs.

De nombreux jeunes chercheurs sont impliqués dans ce programme, et proviennent de disciplines diverses: biologie, archéologie, sciences du goût, psychologie, économie, climatologie, sciences du sport, physique, géologie... Ils livrent tous leurs « histoires de science ». En organisant ces rencontres avec les acteurs de la recherche en train de se faire, l'objectif est de montrer au public que la science est avant tout une activité humaine.

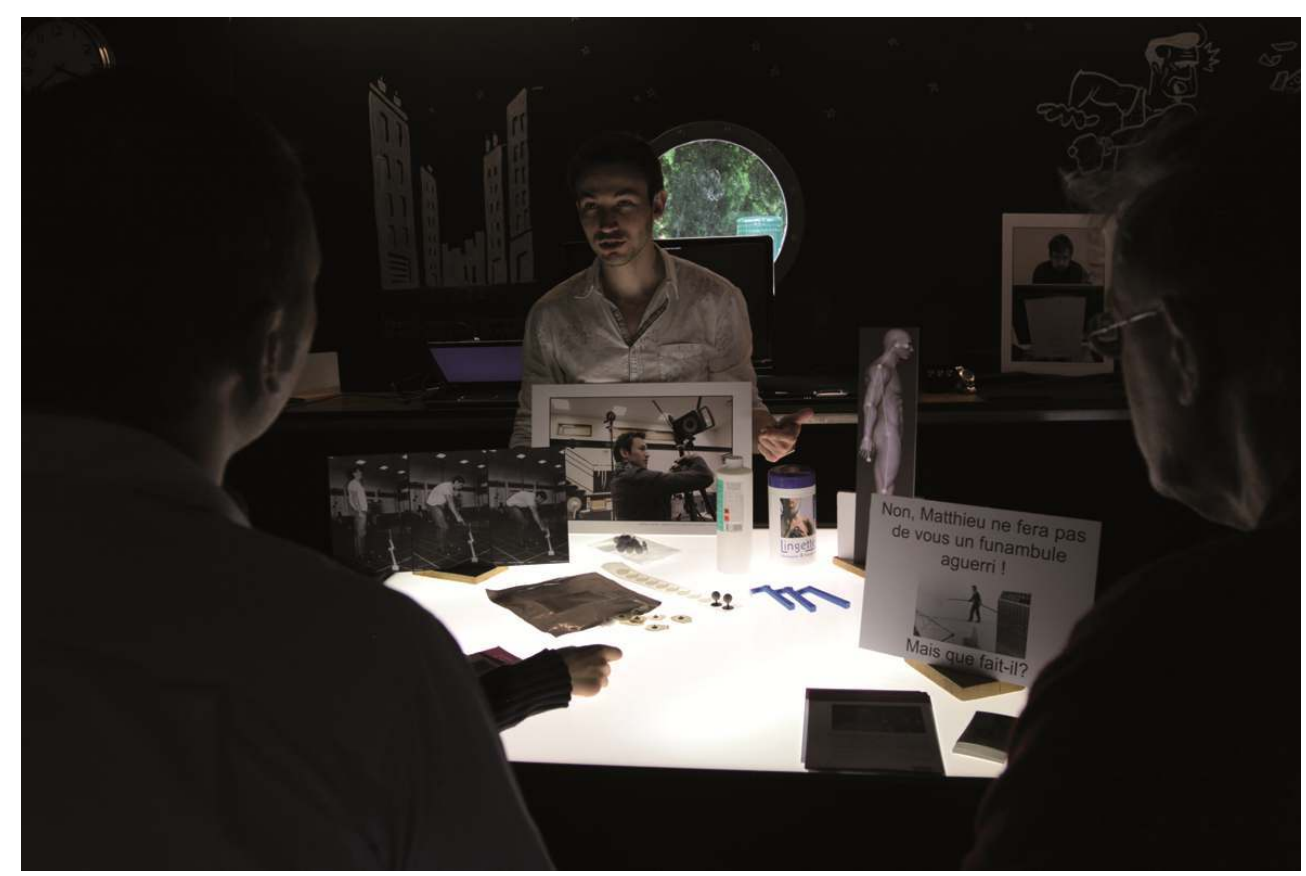

Matthieu Casteran présente sa thèse Le vieillissement et le contrôle des mouvements lors d'un aprèsmidi Experimentarium sur la Péniche Cancale, à Dijon (juin 2012).

(c) Experimentarium

\section{L'Experimentarium délocalisé : mode d'emploi}

La réussite d'une rencontre entre un chercheur et un public en situation de loisir ne va pas de soi. Voici quelques conditions nécessaires sur lesquelles nous travaillons pour adapter l'Experimentarium à différentes situations telles que la rencontre dans une bibliothèque ou la réception d'une classe dans un lieu non-spécifique.

\section{Un chercheur entraîné}

A priori, un chercheur qui vulgarise peut être incompris. Son sujet et son vocabulaire sont souvent complexes, mais la distance avec le public peut aussi venir de questions de sens : pourquoi me raconte-t-il ça ? où veut-il en venir ? à quoi ça sert? 
Prenons le cas des conférences. Souvent, le public est poli et ne fait guère de reproches au chercheur qui a fait l'effort de lui parler. De plus, beaucoup de gens pensent que ces propositions « ne sont pas pour eux ». Ainsi généralement, elles n'attirent pas grand monde. Dans tous les cas, il faut prendre en considération la difficulté de l'exercice de vulgarisation qui peut créer de nombreuses mises à distance scientifique-public.

Pour surmonter ces difficultés, avec l'Experimentarium, nous faisons tout d'abord ressentir la philosophie de notre proposition aux chercheurs avec qui nous travaillons. Nous sommes dans le cadre d'une situation de loisir, privilégiant les dimensions culturelles. Situation qui n'est donc pas exclusivement didactique. Il est important de mentionner ce cadre pour engager le chercheur vers des pratiques originales différentes de l'enseignement.

Ensuite, nous proposons un entraînement à tous les chercheurs qui participent à l'Experimentarium ${ }^{1}$. Il se déroule en trois temps : une discussion sur le contexte et le sujet de la recherche, son sens et les méthodes utilisées; un travail sur la construction d'un atelier de discussion de vingt minutes et l'écriture d'une fiche de présentation destinée au public ; une répétition collective entre les nouveaux participants à l'Experimentarium. Enfin, une analyse critique est effectuée lors de la rencontre avec le public.

Cette formation a pour objectif de permettre au chercheur de mieux s'exprimer et surtout de prendre en considération son auditoire. Il s'agit de mettre en place un type de communication où le partenaire de discussion construit aussi ce moment unique qu'est la rencontre. La rencontre ne doit pas être un exposé répété mais une ouverture réflexive toujours nouvelle et enrichissante pour le chercheur aussi.

\section{Petit groupe, temps court, diversité, sécurité}

Nous limitons les « ateliers discussion » de chaque chercheur à vingt minutes. Cette limite a deux intérêts : ne pas laisser trop de place aux longs développements scientifiques qui peuvent perdre l'interlocuteur et permettre au public qui le souhaite, de rencontrer rapidement le chercheur individuellement pour poursuivre la discussion après l'atelier. Nous préférons donc frustrer plutôt que prendre le risque de perdre un public qui n'a pas forcément besoin de rester écouter cette histoire-ci. Enfin, le temps court a comme effet de forcer le chercheur à être synthétique et donc à choisir l'essentiel, à comprendre l'essence de ce qu'il fait.

Le petit groupe est nécessaire pour privilégier la discussion. Ayant testé plusieurs formules, nous en avons conclu qu'il ne faut pas plus de huit personnes dans cette situation. Nous avons aussi compté le nombre d'interventions du public : il intervient en moyenne sept fois dans un atelier. Ce petit comité force le chercheur a regarder les gens à qui il parle et donc voir les réactions exprimées (par leur visage notamment). Il incite aussi à se séparer du mode présentation (type diaporama) qui n'offre pas les mêmes retours réflexifs qu'une discussion.

Pour nous, la diversité est une valeur clé. Il y a tout d'abord une diversité de personnalités parmi les chercheurs qui participent à l'Experimentarium. Par exemple, certains d'entre eux sont plus timides que d'autres et ceci ne doit pas être une 
barrière à leur participation. La communication scientifique n'est pas que l'affaire de showmen!

Ensuite nous tenons à la diversité des sujets abordés. Nous proposons au minimum trois ateliers qui abordent des domaines scientifiques différents. Étant au jour le jour des explorateurs de la recherche à l'université, nous avons envie de faire connaître sa diversité, qui est une richesse. Ce choix est gagnant puisque les principaux retours positifs du public font référence à cette diversité ; ainsi par exemple : « Je ne pensais pas qu'on étudiait cela... ». Nous sommes dans une culture de découverte.

L'observation de la diversité, des points communs entre les sujets et surtout entre les méthodes permet une meilleure appréhension de la démarche de recherche.

Enfin, dans notre communication, nous prenons garde à ne pas mettre les jeunes chercheurs en difficulté. Une étude japonaise portant sur des bars des sciences dont la formule ressemblait fort à l'Experimentarium a conclu que les chercheurs sortaient très réticents de cette expérience ${ }^{2}$

. En fait, les organisateurs avaient demandé à des jeunes chercheurs de se positionner directement face au public sur des questions telles que le rôle de la chimie, les OGM... Ils ont mis de jeunes chercheurs en difficulté, d'où les ressentis négatifs de ces vulgarisateurs débutants. Pour annoncer chaque Experimentarium, nous prenons garde à communiquer sur la recherche en général et sur la découverte de jeunes chercheurs, sans engager de positionnement qui pourrait impliquer un débat d'opinion. À l'Experimentarium, les questions socialement vives sont parfois abordées mais avec beaucoup plus de compréhension mutuelle scientifiques-citoyens que lors de débats contradictoires.

\section{Penser aux bénéfices pour le chercheur}

Les conditions dans lesquelles se déroulent les Experimentarium prennent en compte ce que la situation peut apporter aux chercheurs. Plus généralement, penser au chercheur lors de la conception d'un programme est important pour faciliter son engagement. Une étude anglaise ${ }^{3}$ montre que les institutions ont trop tendance à penser que les chercheurs ne s'engagent pas assez dans les activités de vulgarisation parce qu'ils n'ont pas le temps ou qu'il existe des barrières liées à la nonreconnaissance financière et institutionnelle de ces activités. En fait, les facteurs les plus déterminants sont : l'opinion que le chercheur a sur l'activité de vulgarisation ; la confiance qu'il a en sa propre capacité à vulgariser et l'aide qu'il pourra trouver ; le fait que des collègues en fasse ; et le fait qu'il ait déjà une expérience dans la vulgarisation ${ }^{4}$. Proposer une activité qui est bénéfique pour lui-même est une manière de réussir à engager le chercheur. La plupart des chercheurs qui ont participé à l'Experimentarium répètent l'expérience et poursuivent leur engagement dans la vulgarisation. Ils développent même une attitude plus créative et exigeante vis-à-vis de cette activité.

Penser aux bénéfices implique une prise de conscience de ce qui se joue à moyen terme pour les chercheurs. Ils y trouvent une manière de mieux exprimer leur activité, de la clarifier ; ils se sentent reconnus en tant que chercheurs et le fait de se raconter leur donne du « cœur à l'ouvrage ». De plus, la rencontre avec d'autres 
jeunes chercheurs est fondamentale, il règne un esprit de camaraderie à l'Experimentarium.

\section{Une exigence nécessaire}

Les conditions que nous imposons comme les mises en garde que nous venons d'exprimer ne doivent pas faire penser que la science n'est pas intéressante en soi ou trop complexe à partager. Nous croyons au potentiel attractif de la communication scientifique. Les histoires de science et les scientifiques sont des « bijoux de la culture » à faire partager. Mais l'expérience montre qu'il est très souvent nécessaire de travailler ces histoires « brutes » de science. Ce travail a des effets d'enrichissements importants non-seulement pour le public, mais aussi pour les chercheurs.

Les quelques conditions nécessaires à la rencontre citées ci-dessus ne doivent pas constituer un cadre trop rigide. Nous avons testé plusieurs « greffes » de notre formule Experimentarium avec des festivals de conte, des visites de laboratoires, des soirées « science et fiction ", des mises en regard avec des propositions théâtrales et très souvent la symbiose a fonctionné. L'Experimentarium a une identité, mais il grandit aussi de ses expériences.

Lionel Maillot

Chargé de médiation scientifique à l'université de Bourgogne Responsable de l'Experimentarium lionel.maillot@u-bourgogne.fr

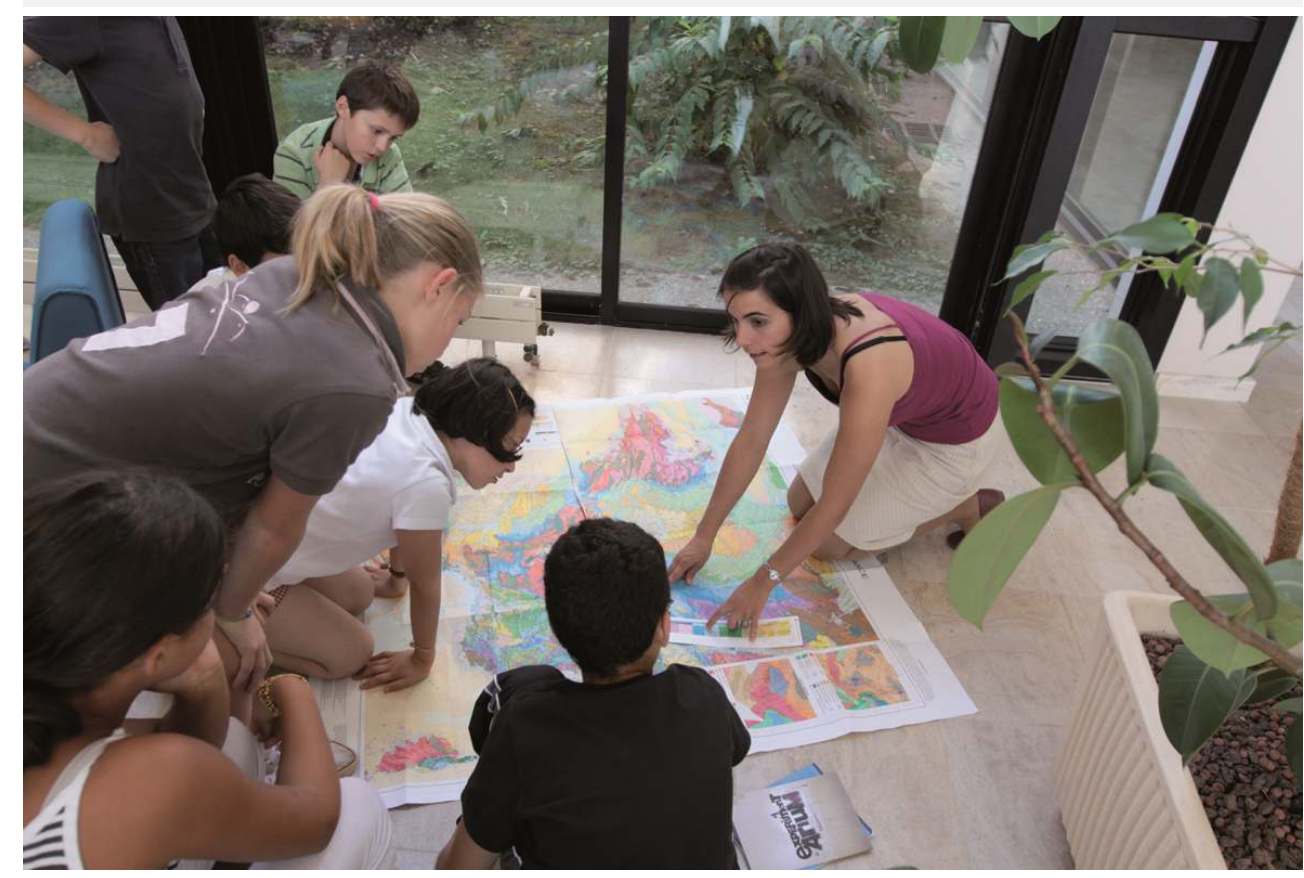

Séance de l'Experimentarium au Centre des Sciences du Goût à Dijon : présentation par Aurélie Bonin de son travail sur Les climats du passé imprimés dans les fossiles (octobre 2011).

(c) Experimentarium 


\section{Partenariat avec les bibliothèques municipales}

3 Cinq semaines par an, l'Experimentarium accueille des scolaires et le grand public sur le campus dijonnais. Ainsi chaque année, nous recevons une quarantaine de classes et les journées «Grand Public » attirent plusieurs centaines de personnes. Cependant, si nous voulons que ces évènements de rencontre avec des chercheurs puissent être accessibles à tous, il nous semble pertinent de sortir de l'enceinte de l'université et d'aller à la rencontre du public en investissant de nouveaux lieux, des espaces faisant partie du quotidien des habitants d'une ville. L'Experimentarium devient alors «nomade» et s'installe quelques jours par an dans d'autres lieux (sous les halles du marché de Dijon ou dans d'autres villes de la région par exemple) afin de toucher un public différent de celui qui fait la démarche de venir sur le campus. C'est avec cet objectif d'ouverture que l'Experimentarium et les bibliothèques municipales de Dijon se sont associés en 2009 pour initier un dispositif de dialogue entre les chercheurs et les lecteurs des bibliothèques. Au cours d'un après-midi, au milieu des rayonnages, le public découvre les chercheurs et échange avec eux. Ces rencontres permettent de promouvoir la culture scientifique et de dynamiser les activités des bibliothèques et de l'Experimentarium. Chaque année, ces événements ont lieu dans quatre bibliothèques de Dijon et de Chenôve (ville voisine).

\section{La parole au milieu des écrits}

Amener des chercheurs dans une bibliothèque et organiser des rencontres avec le public ne se limite pas à investir un nouveau lieu, s'installer entre les murs d'un quelconque bâtiment pour organiser un évènement de culture scientifique. Au contraire l'identité, le fonctionnement et l'ambiance de ce lieu sont importants.

Dans une bibliothèque, on chuchote. Nous avons pu observer que le fonctionnement plutôt silencieux d'une bibliothèque influait sur les échanges entre le public et les chercheurs. En effet le chercheur s'installe au milieu des rayonnages. Il pose sur une table des objets, des photographies et des illustrations de son quotidien de recherche. Le public, parce que l'espace n'est pas très grand, parce qu'il ne faut pas parler trop fort pour ne pas déranger les autres lecteurs, doit se regrouper autour du chercheur pour écouter ses histoires de science. Tout ceci crée une certaine complicité, une intimité qui favorise l'échange. Le nombre de participants à un Experimentarium dans une bibliothèque est plus faible que sur le campus ou bien sous les halles du marché dijonnais. Mais ce lieu offre des avantages en terme de proximité entre les chercheurs et les personnes venues les rencontrer. 


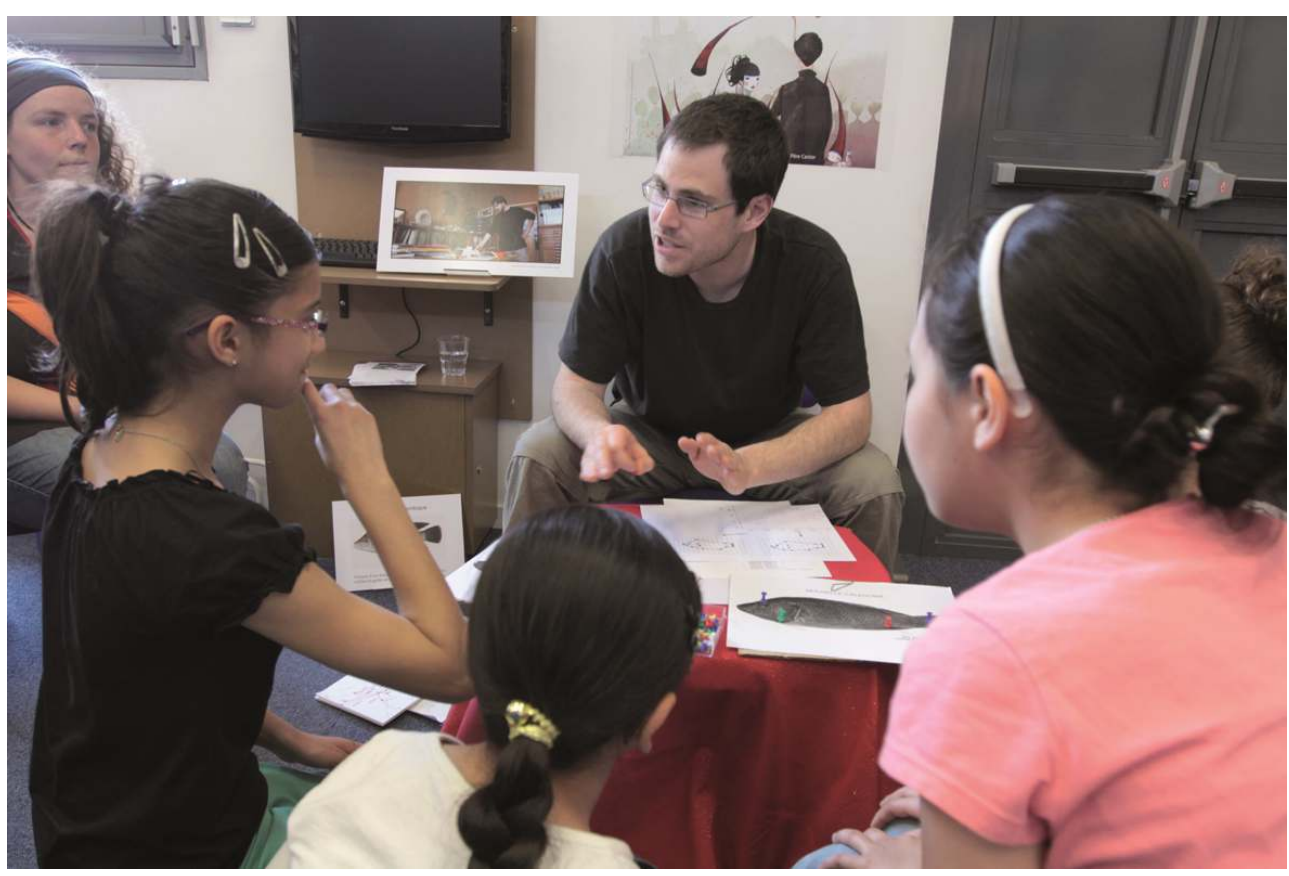

Cyril Firmat présente sa recherche sur L'évolution d'une espèce invasive à la bibliothèque municipale de Chenôve (mars 2012).

(c) Experimentarium

Les jeunes chercheurs, installés au milieu des livres, partagent leurs connaissances avec le public, dévoilent leur quotidien de recherche, leurs espoirs, leurs doutes, leurs découvertes. C'est un instant de témoignage et de diffusion des connaissances, un instant à l'image de la bibliothèque, ce lieu « miroir » des hommes et du monde ${ }^{5}$. C'est en cela que l'identité du lieu est importante. La bibliothèque est un lieu vivant, riche de par la diversité de ses ouvrages, des histoires et des savoirs qu'ils contiennent. Ce cadre offre les conditions propices aux échanges, à la transmission et à la découverte.

\section{Le livre, la science et le lecteur}

7 La diversité des ouvrages d'une bibliothèque peut être importante. On peut s'interroger sur la place que la science occupe au sein des rayonnages. Des journées d'études ont été consacrées à cette question ${ }^{6}$. Le constat semble être le même : les sciences et techniques occupent souvent peu de place dans les collections des bibliothèques. Est-ce le budget alloué qui fait défaut, ou bien le fait que les bibliothécaires ont, le plus souvent, une formation plus littéraire que scientifique ? À moins que ce fait ne traduise des doutes sur le lectorat potentiellement intéressé par ces ouvrages.

Or les bibliothèques jouent un rôle important dans la transmission culturelle qui comprend également la culture scientifique. C'est dans ce sens que le partenariat entre des bibliothèques de Dijon (et alentours) et l'Experimentarium s'est initié et perdure depuis quelques années. L'expérience d'organiser des rencontres avec des chercheurs de l'université autour de leurs travaux en cours, nous montre qu'il existe un public de lecteurs intéressé par la recherche, ou tout du moins par ses acteurs.

9 Rappelons que lorsque nous parlons de culture scientifique, de recherche en cours ou de science plus généralement, nous incluons dans ces termes les sciences humaines. À chacune des manifestations de l'Experimentarium en bibliothèque, des chercheurs en 
sciences humaines et sociales sont présents: psychologie, philosophie, sciences de l'éducation, économie, archéologie... Ces disciplines font partie intégrante de la recherche actuelle et le public peut le constater à chaque manifestation de l'Experimentarium.

Avec l'essor du Web, nous sommes à la fois presque noyés et souvent seuls sous le flot des informations disponibles. Le gigantisme du nombre des sites Internet offre un nouvel accès aux connaissances, mais comment trier le bon grain de l'ivraie? Démocratie du savoir ou désinformation? Une chose est sûre : avec l'essor du numérique la relation au livre change, tout com-

11 me évolue la relation du public vis-à-vis de la science. Tout ceci ne remplace pas la médiation. L'objectif en organisant ces rencontres avec le public, n'est pas de mettre le chercheur sur un piédestal, mais de donner l'opportunité à tous de lui poser directement des questions, de discuter, de soutenir ou de critiquer ses travaux en cours, de mieux connaître ce monde parfois inconnu qu'est la recherche. Il faut également garder à l'esprit que les bénéfices sont réciproques et que les chercheurs, en sortant de leurs laboratoires, en se confrontant au public, ont beaucoup à gagner de ces rencontres.

Dans l'avenir, il nous semblerait important d'accentuer le lien entre le discours du chercheur et les livres qui l'environnement lorsqu'il rencontre les lecteurs d'une bibliothèque. Ceci permettrait peut-être de mettre en valeur les collections d'ouvrages liés à la science, de susciter l'intérêt du public et des bibliothécaires.

\section{Pas si simple de faire simple}

13 Il ne faudrait pas croire qu'amener des chercheurs dans une bibliothèque afin qu'ils discutent de leurs travaux avec le public est une tâche simple. Pour que la rencontre se passe le mieux possible, tant pour les chercheurs que pour les visiteurs, une préparation en amont est obligatoire et une certaine exigence est nécessaire. L'objectif n'est pas de "faire aimer la science", de susciter des vocations chez les plus jeunes, ni de glorifier tous les travaux de recherche en cours, mais plutôt de faire se rencontrer deux partenaires que l'Histoire a souvent éloignés. L'exercice est d'autant plus délicat que la vulgarisation scientifique, si elle est mal faite, peut au contraire couper, parfois définitivement, les liens déjà fragiles entre le public et le monde de la recherche (voir l'encadré « L'Experimentarium délocalisé, mode d'emploi »).

\section{Culture et culture scientifique?}

14 Se rendre dans une bibliothèque, tout comme aller dans une salle de spectacle ou un musée constitue une pratique culturelle. La rencontre avec des chercheurs devrait, de notre point de vue, l'être également. Or nous pouvons constater que c'est rarement le cas, qu'une distinction s'est opérée entre la culture (artistique, littéraire, humaine) et la culture scientifique. Nous pouvons reprendre l'allocution de Jean-Marc Lévy-Leblond lors de l'inauguration de l'ISEM (Institute for scientific methodology) de Palerme en mars 2007: «L'idée même qu'il puisse exister deux cultures me parait contradictoire : le mot culture ne peut être pensé qu'au singulier; comme la République française, la culture est une et indivisible. Que serait une culture fragmentée, sinon justement, une non-culture? Ce qui constitue la culture en tant que telle, c'est précisément sa capacité d'exprimer et de développer des liens organiques entre toutes les dimensions de l'activité humaine. » 
L'auteur nous invite à reconnaître le caractère culturel de toute activité de connaissance (l'activité scientifique comprise), et à récuser une dichotomie entre le monde des faits scientifiques et le monde des cultures humaines.

Organiser un Experimentarium dans ce lieu culturel qu'est la bibliothèque, va dans ce sens d'une unicité des cultures humaines. Médiateurs scientifiques, chercheurs et bibliothécaires peuvent travailler ensemble dans cette direction afin que la culture scientifique se « (re)mette en culture ».

\section{NOTES}

1. Nous proposons des stages de formation pour des universités, écoles doctorales ou organismes de recherche : http://experimentarium.u-bourgogne.fr/spip.php?rubrique50

2. Mizumachi, E. et al. Scientists' attitudes toward a dialogue with the public: a study using "science cafes", Journal of science communication, novembre 2011.

3. Poliakoff, E. et Webb, T.-L What Factors Predict Scientists' Intentions to Participate in Public Engagement of Science Activities? Science Communication, décembre 2007.

4. Maillot, L. L'engagement des chercheurs dans la vulgarisation, Actes des Journées Hubert Curien, 2012.

5. Nous faisons référence à l'ouvrage Figuier, R. La bibliothèque : miroir de l'âme, mémoire du monde, Autrement série Mutations, $\mathrm{n}^{\circ} 121,1991$.

6. Nous faisons référence aux journées d'études organisées par (ou en collaboration avec) l'association des bibliothécaires de France (ABF) comme : le 4 décembre 2006 à Tours sur le thème "Sciences en bibliothèque ", le 7 octobre 2010 à Montbéliard sur le thème "Quelle place pour les sciences en bibliothèque, " et le 25 octobre 2012 à Dijon sur le thème " Lecture publique et culture scientifique ».

7. Lévy-Leblond, J.-M. (Re) mettre la science en culture : de la crise épistémologique à l'exigence éthique. Courrier de l'Environnement de l'INRA, n56, 2008, pp. 7-16.

\section{RÉSUMÉS}

Le travail de médiation de la recherche mené auprès du grand public par l'Experimentarium de l'université de Bourgogne comprend depuis peu un développement au sein des bibliothèques de lecture publique : l'auteur montre les apports de cette expérience et en particulier le climat de complicité ainsi créé entre les jeunes chercheurs et le public. 
AUTEUR

\section{ÉLISE CELLIER-HOLZEM}

Élise Cellier-Holzem est chargée de l'Experimentarium à l'université de Bourgogne. elise.cellierholzem@u-bourgogne.fr 